\title{
Argumentative Skills as a Basic Component of Professional Development of Future Teachers
}

\author{
Fauziya Sametova ${ }^{1,}$, Enipa Muktarova², Nazym Ozekbayeva ${ }^{3}$, Assima Ryskulbekova $^{4}$, \\ Kairbek Alimbetov ${ }^{5}$ \\ ${ }^{1}$ Department of Philological Sciences, Kazakh National Women’s Teacher Training University, Kazakhstan \\ ${ }^{2}$ Department of Pedagogical Sciences, Kainar Academy, Almaty, Kazakhstan \\ ${ }^{3}$ Department of Pedagogical Sciences, Kazakh Medical University of Continuing Education, Kazakhstan \\ ${ }^{4}$ Department of "Pedagogy and Methodology of Primary School", The National Pedagogical University named after Abai, Kazakhstan \\ ${ }^{5}$ Department of Languages and Journalism, Kainar Academy, Kazakhstan
}

Received August 14, 2020; Revised October 24, 2020; Accepted November 4, 2020

\section{Cite This Paper in the following Citation Styles}

(a): [1] Fauziya Sametova, Enipa Muktarova, Nazym Ozekbayeva, Assima Ryskulbekova, Kairbek Alimbetov, "Argumentative Skills as a Basic Component of Professional Development of Future Teachers," Universal Journal of Educational Research, Vol. 8, No. 12, pp. 7175 - 7182, 2020. DOI: 10.13189/ujer.2020.081284.

(b): Fauziya Sametova, Enipa Muktarova, Nazym Ozekbayeva, Assima Ryskulbekova, Kairbek Alimbetov (2020). Argumentative Skills as a Basic Component of Professional Development of Future Teachers. Universal Journal of Educational Research, 8(12), 7175 - 7182. DOI: 10.13189/ujer.2020.081284.

Copyright $@ 2020$ by authors, all rights reserved. Authors agree that this article remains permanently open access under the terms of the Creative Commons Attribution License 4.0 International License

\begin{abstract}
The modern period of educational development characterizes various attempts in higher quality and adequate result provision. Having acquired the modernized educational paradigm, our academic community placed personality development through communication under the focus of primary research. The relevance of the problem is that those future teachers are required to conduct a dialogue. The competency-based concept claims for professionally ready specialists who are capable of arguing in the academic field. The purpose of the article is to develop the ability to conduct reasoned discourse. In this sense, and under the circumstances of the current development of Kazakhstani society, it is inevitable to form the ability to conduct argumentative discourse as a crucial element for future teachers. Nowadays, modern foreign language teachers must have basic language knowledge and skills and upgrade teaching methods and techniques. That is why modern foreign language teachers need to develop intercultural communicative competence using all types of dialogical speech. There is the necessity of investigating the terms "discourse" "argumentative discourse" to create argumentation methods. This article examines the theoretical aspects of investigations on the themes discourse. Also, there is a summary of the argumentative discourse investigations as one of the available kinds of oral discourse.
\end{abstract}

Keywords Argumentative Discourse, Skills, Functions of Language, Speech Acts, Teaching Process

\section{Introduction}

It is already much said about what a teacher should be like and how shethe should behave. However, the debate on becoming more professional, more effective, and economically valuable is still in progress. The day's reality has been raising problems, incorporating the overall social development and its reflection in younger generations. Modern communicational behavior and discussion styles have undergone crucial changes due to diverse factors, including economic, political, and cultural factors. Unfortunately, not all changes in communication models accepted have positively affected the overall social interactions. Quite a long observation within educational institutions demonstrates a lack of elementary elements of culture while defending their points of view, leading to severe conflicts. Many people believe that today's teacher's significant role is to prepare young specialists for real professional life as communicative behavior. However, the situation reveals the sad truth that the right is the one who 
cries louder or has more authority. That is why developing the argumentation culture is the perfect tool for overcoming this educational issue and 'curing' the nation. By implementing argumentative discourse skills development, we will raise the general status and equip students with the necessary argumentation techniques in the academic field. Thus, the article is primarily focused on the necessary background of argumentative discourse design, which would be beneficial within the educational process.

Argumentation, as a scientific category, has been in the focus of investigation interest since ancient times. Today, particular attention to the issue is paid, especially by philosophic, logic, and rhetorical studies. No doubt, each discipline underlines definite peculiar features and identify its approaches to argumentation research. Within the framework of the modern educational paradigm of education, we are to prepare competent specialists to realize and solve problematic professional tasks. Concerning teachers, our main 'sword' is a language, how we operate it, and how others assume our intentions. In this sense, the concept of argumentative discourse culture is seen as the adequate means for educational purposes, and its awareness might be interpreted as the power to establish rapport with interlocutors and gain a consensus in the process of communication. Some scholars point out that communication is a process through which an addresser intends to modify an addressee's cognitive environment. Each communicative act presupposes a certain degree of cognitive influence; that is why engaging in communication presupposes mastering specific argumentative techniques to achieve a particular goal. Communication, then, is not a mere coding-decoding process, but, above all, an inferential process, that is, a reconstruction of the addresser's message, which gives. As a result, the broader coincidence of both cognitive environments.

In the communicative process, culture plays three roles. Modern school is intended to join future generations to the culture of the native country, to cultivate universal human values in them. To develop a tolerant attitude to the culture of the countries of other peoples and the ability to participate in cross-cultural dialogue [1, P.149-153]. Firstly, it is from and through the communicators' cultural schemata that the communicative situation is perceived and understood, and the communicative act is created. Secondly, it is also from and through the communicators' cultural schemata that the meaning of the addresser's communicative act may be inferred. Furthermore, finally, the communicative act's result is a modification of the communicators' cognitive schemata. Thus, culture plays a crucial role in communication and may be regarded as a vital element for argumentative skills formation. However, displaying a culturally appropriate communicative behavior does not promote persuasive argumentation on its own.

\section{Findings}

The advantage of a discourse-based model is that it acknowledges social interaction's role in constructing the argument. Only in very formal settings, such as courtroom proceedings or political debates, are arguments presented outside of a conversational context. Most often, arguments arise from disagreements people have with one another. Arguments are likely to be initially incomplete and to grow as the speaker addresses the challenges presented by a conversational partner. V. Grigorieva [2] supported this claim by showing that arguments may be logically sound even if they are incomplete by formal logic; that is, an argument may be valid even though its underlying premises remain implicit.

Furthermore, individuals may not elaborate arguments unless they recognize the need to clarify them or convince their audience. Grice's (1975) maxim of quantity holds that a speaker will provide only as much information as necessary for an audience to construct meaning. Thus, discourse is integral to the construction of an argument. If this is the case, then the best way to examine the development of argumentative competence is to examine the process by which individuals construct arguments in the context of discourse.

If we regard argumentative discourse as an activity in development, two forms of development can be identified. One is enhanced skill in directing the course of critical dialogue to meet the activity's objectives. The other is an enhanced understanding of the goals of argumentative discourse. These two forms of development, we predict, reinforce one another. In other words, progress in strategic performance is propelled in part by a better understanding of discourse goals. Simultaneously, the exercise of these strategies in discourse activity promotes a more refined understanding of the activity's goals as proposed in other strategic cognitive development areas by A. Weston [3]; metalevel understanding both directs and is informed by strategic performance.

To understand the conditions under which argumentative dialogue promotes reasoning, it is critical to consider people's goals while arguing. In argumentative dialogue, one can distinguish two overlapping but distinct kinds of activity: dispute and deliberation (Kroll, 2005). Both kinds of discourse involve two or more speakers who contrast alternative viewpoints by evaluating claims and the evidence used to support them. However, disputes and deliberation can be distinguished by their goals [4, P.34-61]. In a dispute, the goal is to defend a viewpoint and undermine alternatives, whereas, in deliberation, the goal is to arrive at a viewpoint by comparing and evaluating alternatives. These diverging goals, in turn, create essential differences in the social dynamic between conversational 
partners. In the dispute, participants compete intending to persuade others to adopt their opinion. In deliberation, participants collaborate intending to work towards a consensus view.

These discourse activities, dispute, and deliberation, in turn, may impact how individuals process opposing viewpoints. As Leitao (2009) points out, the process of negotiating viewpoints can prompt an array of responses from an individual. When speakers confront opposing claims and evidence in argumentative dialogue, they have at least four primary responses at their disposal: to dismiss counterarguments and maintain their position; to agree with counterarguments locally, but deflect their impact by turning to other claims in support of their position; to integrate counterarguments by qualifying or adjusting their position; to accept counterarguments and abandon their position.

When consensus is the goal of dialogue, individuals allow themselves the full range of these responses. In contrast, when persuasion is the goal of dialogue, individuals must dismiss or deflect counterarguments to convince others to adopt their conclusions. Thus, clear goals in discourse may limit the value of argumentative dialogue for reasoning by constraining the options that individuals believe they have for responding to alternative viewpoints. As a result, the constraints of discourse goals while arguing may lead individuals to process opposing side claims and evidence superficially. To prevent the dismiss of the argumentation process, each communicative act should be appropriately organized.

An argument's nature is nearly two-fold: there is an underlying statement of purpose or claim, the very point presented (for questioning or dispute), and exposed for acceptance. The supporting evidence will be used as grounds or reasons to support the validity of the claim. A third and necessary ingredient to a compelling argument is the linkage between the claim and the supporting evidence, the component that ensures that the evidence is, in fact, directly related to the validity of the claim. This refers to the warrant, and for an understanding of this component, we need to credit the work of F. Saez [5, P.103-120].

The strategy essentially questions the relation of each piece of evidence related to the claim's overall credibility. In looking at the structure of arguments, it is vital to account for the connections between the reasons offered for the support and the claim's nature. If the connection is not there, the claim can lose its value. This model of argument construction is the most appropriate for educational purposes. Thus, structuring each communicative intention following this model's principles, an addresser (a future specialist in business communication) is believed to gain the most effective result. However, in particular, any discourse and argumentation are presented by the cohesive and coherent text. There are various tools for creating formal and semantic connections between utterances within the discourse. Typically, they are differentiated between four broad groups: grammatical (syntactical): word order, tense forms unity, aspects of verbs, conjunctions, parenthesis, semantic: repetition; logical: cause-effect relations, conditions, clauses; pragmatic: communicative act inference via presuppositions.

\section{Methodology}

Our cognitive linguacultural methodology presents four stages of the above-mentioned sub competences formation in teaching three languages. Each stage fulfills its specific functions and sets individual skills to form on each stage, which equips by the most effective methods and techniques. Moreover, each stage must be equipped with particular means of teaching with various visual Internet resources that the teacher can use during the lesson. There should be a mention that visualization plays a significant role in what we call cognitive learning. It can also give the learners a chance to perceive the necessary material and interpret the conveying message's meaning expressing their creativity and individuality.

In order to develop argumentative skills in future specialists, we offer the stages. The first two stages of our educational model (perceiving basic notions and accumulating relevant data) are equipped with thematic units, which present in the form of text to read or track to listen to them. However, teachers can vary an organization of data perception with the help of video. The advantages of using videos in the first two stages are the following: it is more action-packed and eventful than reading or listening. It helps to keep your students attentive; the most important fragments of information that need to be memorized are usually accompanied by voice shades and different facial expressions, facilitating memorizing necessary material. The third stage is a stage of quasi-communication. It characterizes implementing images, videos, and speech paradigms. The quasi-communication stage presupposes the modeling of typical communicative business situations. In other words, students prepare to free, spontaneous communication by role-playing typical communicative acts with the help of speech clichés. Students train both monologue and dialogue speech; the communicative tasks convey means of images and video tracks. The video offered to watch the students, and afterward, the teacher provides them with a set of communicative tasks to fulfill. To provide an example, we have chosen the video track that shows students the E-tandem method of argumentation. After the track is shown, the students are offered the following tasks: express your opinion about the advantages and disadvantages of E-tandem argumentation; discuss with the partner the specificities of organizing E-tandem, and share opinions about the ways partners can overcome communication difficulties. Write an argumentative essay discussing the advantages and disadvantages of oral (telephone, video conferencing, etc.) and written (e-mail, 
letters, instant messaging, etc.) E-tandem [12]. Speech paradigms can be used in the third quasi-communication stage. Speech paradigms can be defined as handouts that provide learners with vital speech clichés (in case classes are taught in a foreign language) that help them sound natural and be aware of native speakers' starting/continuing/concluding norms speech in different situations. In other words, they can be defined as linking words and phrases that are grouped for usage in a particular case (discussion, argument) and presented in a compact form to be a constant helper for a learner till he/she memorizes it well. This stage prepares students for the next stage of free communication, where they should be able to participate in a free, spontaneous discussion, debate using arguments.

On the stage of free communication, the case study could be implemented.

The term 'case study' covers a wide range of analysis problems, but most types include several key elements. Most cases are based on real or constructed events, which could reasonably take place. They tell a story, one involving issues or conflicts needed to be resolved - through most case studies, do not have one obvious or clear solution. The information contained in a case study might be complex (including charts, graphs, and relevant historical background materials) or simple - a business story that illustrates a problematic situation requiring a decision [13]. Case studies are involving real characters in actual situations. A good case presents an interest-provoking issue and promotes empathy with the central characters.

Case studies usually generate animated class discussion, especially if students feel that the case will serve as a basis for wide-ranging exploration. Instructors can emphasize that the analysis will be a group project and that no one will be criticized for raising naive questions or uncertainties. The group must recognize the significance of cooperation in making sense of the problem and that everyone must work together on the analysis actively. Without a clear sense that they are free to experiment with hypotheses, students will tend to remain silent until they feel that the 'right' answer has been identified. Students develop analytical thinking and argumentation skills by discussing complex, real-life scenarios [14]. The case method is a student-centered, highly interactive pedagogy that changes the classroom process. The case study illustrates many features that permit us to treat it as the most effective techniques that should be used as a key for argumentation acquisition. It is based on real events (or describes events that can take place); it contains implicit information that needs to be investigated; it presupposes an open-ended solution (or several ones); it develops collaborative skill; it lets students express their creativity.

Problem-based learning can also be used on the stage of free communication. It consists of carefully designed problems that challenge students to use problem-solving techniques, self-directed learning strategies, team participation, and argumentative skills. Students work with classmates in problem-based learning courses to solve complex and authentic problems that help develop content knowledge and problem-solving, reasoning, argumentative communication, and self-assessment skills. These problems also help maintain student's interest in course material because students realize that they are learning the skills needed to succeed in business communication. Problem-based learning is characterized as a student-centered approach, teachers as "facilitators rather than disseminators," and open-ended problems serve as the initial stimulus and framework for learning. Instructors also hope to develop students' intrinsic interest in the subject matter, emphasize learning instead of a recall, promote group work, and help students become self-directed learners. The students are given the freedom to study those topics that interest them the most and determine how they want to study them. Students should identify their learning needs, help plan classes, lead class discussions, and assess their work and classmates' work.

Students develop a deeper awareness and ownership of essential concepts by working on activities, a basic tenet of the constructive learning approach. Students must learn to be conscious of what information they already know about the problem, what information they need to know to solve the problem, and the strategies to solve the problem. It helps students to become more effective problem-solvers and self-directed learners. However, many students are not capable of this sort of thinking on their own. For this reason, the instructor must become a tutor or "cognitive coach" who models inquiry strategies, guides exploration and helps students to clarify and pursue their research questions. The instructor plays a critical role in helping students to become self-directed learners and must create a classroom environment in which students receive systematic instruction in conceptual, strategic, and reflective reasoning in the context of a discipline that will ultimately make them more successful in later investigations.

The first-class meetings in problem-based learning include brainstorming sessions in which issues central to the course are identified. Alternatively, the instructor can create an extensive list of topics and ask students to focus on those topics that seem most interesting. Based on student input about course topics, the instructor develops ill-structured problems. Students then work on the problems in groups of three to eight students, depending on the number of students in the course and the number of available instructors or tutors. Regardless of how selected topics, the instructor presents student groups' problems before providing any formal instruction on the topic. However, problems are introduced with "mini-lectures" that provide some context for the problem and identify potential difficulty areas.

During class time and outside of class, students work with their group to solve problems. Throughout each class, 
the instructor must ensure that all students are involved in the problem-solving process and familiarize them with the resources needed (e.g., library references, databases) to solve the problems and identify common difficulties [15, P.241-249]. With multiple groups exploring different problems or examining similar problems, coaching groups' tasks may be too much for one instructor [16, P.331-334].

Thus, the instructor may want to consider using teaching assistants or tutors familiar with this method and techniques to assist groups. Upon completing the research or inquiry phase of problem-solving, groups may be required to write the arguments and present them to the rest of the class.

\section{Results}

In experimental groups, the learning course and content were formed in accordance with four stages and a set of exercises. At the final stage, the students had to present an independent research project. The work was carried out in mini-groups of 3-5 people, where each group was engaged in implementing the idea that had been suggested by previous tasks. The project work was realized with the help of a few stages, which included planning where the discussion of the topic in mini-groups took place to determine the research work direction. The project work's organizational stage determined the periods for the project implementation and each of its sections, methods of the research work, and allocation of the students' responsibilities within mini-groups. During the analytical stage, the students realized the topic's independent research activity using different data collection: work with unique literature, the Internet, students' personal experience, consultation with experts, observation, questioning, interview, and mass media analysis.

At this stage, the analysis of the information received has been conducted. Each student created a blog of his topic, which served as his personal diary. During the work under the project; each student kept his blog to analyze the work and presentation of the exciting facts he found discussing the problem with interested users. The next stage is a stage of information consolidation. The students used their wiki page as a kind of "canvas" for a collective work, where each of them posted necessary information, corrected and explained the information already added. The result of this work is a collective article on the topic of the project available on the Internet and, at the same time representing the content of the project. At this stage, the students could apply their creative skills to select the most optimal method of presentation, which could have different formats: screen, brochure, video.

After that, the team had to defend their project, providing arguments. A post-experimental diagnostic of the level of argumentative skills development was carried out in experimental and control groups. The results of the post-experimental diagnostics were based on mathematical data processing. The dynamics of skills development in experimental and control groups were notably different. The students from experimental groups have demonstrated the improvement of all testing parameters, while the students from control groups have not demonstrated significant improvement in the tested skills.

In order to develop argumentative skills in future teachers, we offered the stages. The first two stages of our educational model (perceiving basic notions and accumulating relevant data) were equipped with thematic units, presented in the form of text to read or track to listen to. The third stage has presupposed the modeling of typical communicative situations. In other words, students were being prepared to free, spontaneous communication by role-playing typical communicative acts with the help of speech clichés. Students trained both monologue and dialogue speech; the communicative tasks were conveyed through images and video tracks. Students were offered to watch the video, and afterward, the teacher provided them with a set of communicative tasks to fulfill. The case study, problem-based learning, and project work could be implemented on the stage of free communication. Experimental verification of the proposed model had been conducted based on Kainar Academy in the university's natural learning environment following the schedule has been given. We have selected two groups (experimental and control). During the planning stage of the experimental work, we have differentiated variable and non-variable dimensions. The model proposed by us and constructed using innovative communication technologies was a variable dimension in the experimental work. The control group students had the traditional classes of scholarly communication while the students of experimental groups had classes based on presented stages. The task system developed by authors and the number of hours were non-constant measurements.

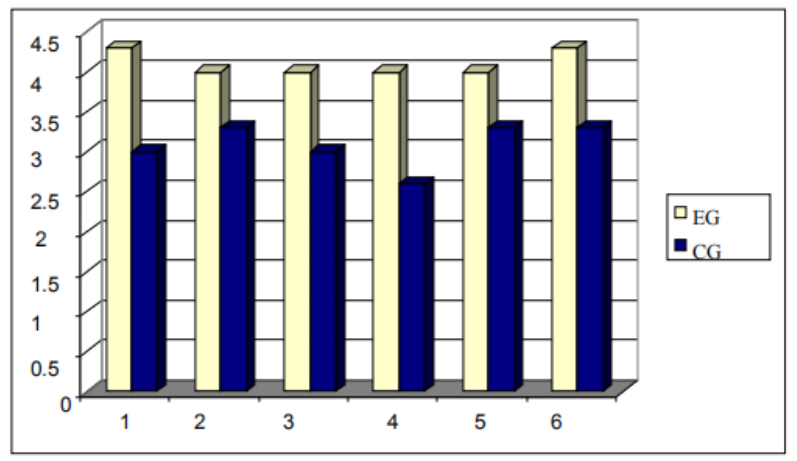

1. Ability to convince and assert addressee by means of a language. 2 . Ability to accept positions and circumstances and reflect it with the help of a language. 3. Ability to give clear directions and convince in the necessity to follow them. 4. Ability to express (personal) opinion and convince addressee in its relevance. 5. Ability to propose new positions using a language. 6. Ability to reflect the positions in argumentation.

Figure 1. The post-experimental results (experimental group EG and control group CG) 


\section{Discussions}

Some linguistic theories have attempted to separate the mental knowledge of language from language use. In Chomsky's terms, this is the distinction between competence (knowledge) and performance (use). Chomsky privileges competence over performance as the subject matter of linguistics [6, p. 50]. In rejecting the distinction between competence and performance, cognitive linguists argue that knowledge of the language is derived from language use patterns, and further, that knowledge of the language is knowledge of how language is used.

In the words of psychologist and cognitive linguist Michael Tomasello [7, P. 721-727], language structure emerges from language use. This is known as the usage-based thesis. It follows from the assumption that language structure cannot be studied without considering the nature of language use. This perspective is what characterizes argumentative discourse as a functional rather than a formal approach to language. Perhaps the most crucial concept of the usage-based approach is utterance. An utterance is a particular, actual occurrence of the product of human behavior in communicative interaction, as it is pronounced, grammatically structured, and semantically and pragmatically interpreted in its context [8]. An utterance is a linguistic act in which one person expresses towards another, within a single intonation contour, a relatively coherent communicative intention in a communicative context. As these statements indicate, an utterance an instance of linguistic behavior on the part of a language user. A language user is a member of a particular linguistic community who attempts to achieve a particular interactional goal or set of goals using particular linguistic and non-linguistic strategies. Interactional goals include attempts to elicit information or action on the hearer's part, provide information, and establish interpersonal rapport.

The linguistic strategies employed to achieve these goals might include speech acts (requesting, informing, promising, thanking, and so on). Non-linguistic strategies include facial expressions, gestures, the speaker's orientation, interpersonal space, and so on. However, an utterance is not a discrete or precisely identifiable unit. This is because utterances involve grammatical forms (word order), semantic structures (patterns of the meaning) speech sounds, intonation patterns, slight pauses, and accelerations and decelerations.

In this respect, utterances differ from the related notion of a sentence. A sentence, as defined by linguistics, is an abstract entity. Utterances typically occur spontaneously and often do not conform to the grammaticality requirements of a well-formed sentence. For example, in terms of structure, an utterance may consist of a single word, a phrase, an incomplete sentence, or a sentence that contains errors of pronunciation or grammar because the speaker is tired, distracted, or excited. As this discussion indicates, while a sentence can be precisely and narrowly defined, an utterance cannot. While sentences represent the structure associated with an utterance, utterances represent specific and unique language use instances in business communication. Typically, cognitive linguists place little emphasis on the sentence as a theoretical entity. In contrast, the notion of a usage event or utterance is central to the cognitive perspective.

After outlining the main components of a usage-based view of the language system, in business communication, we focus on two cognitive linguistics areas that attempt to integrate the usage-based thesis with theoretical models of various linguistic phenomena. The first phenomenon is focused on knowledge of the language. In this context, the term 'grammar' is used in its broadest sense to refer to the system of linguistic knowledge in the speaker's mind. In this sense, 'grammar' refers not just to grammatical phenomena like syntax but also meaning. The cognitive model of grammar encompasses the units of language, which constitute the language; and the processes that relate and integrate the various constructions in a language system. The specific theory is called Cognitive Grammar, developed by Ronald Langacker [9].

The second phenomenon we consider is a language change. Here, we examine William Croft's Utterance Selection Theory of language change. This theory views language use as the interface that mediates between the conventions of a language (those aspects of use that make a language stable) and mechanisms that result in deviation from convention resulting in language change [8].

Moreover, in accordance with the aspect of the discourse, it is necessary to highlight the functions of the language and communicative acts laid under the position presentation. In almost all the situations in which we find ourselves, language allows quick and effective expression and provides a well-developed means of encoding and transmitting complex and subtle ideas. These encoding and transmitting notions turn out to be important as they relate to two key functions associated with language, the symbolic function and the interactive function. One crucial function of language is to express thoughts and ideas. That is, language encodes and externalizes our thoughts. The way language does this is by using symbols. Symbols are bits of language. These might be meaningful subparts of words, whole words, or 'strings' of words. These symbols consist of forms, which may be spoken, written, or signed, and meanings with which the forms are conventionally paired. Meaning is the semantic content associated with the symbol. The meaning associated with a linguistic symbol is linked to a particular mental representation termed a concept. Concepts, in turn, derive from percepts.

In teachers' life, language serves an interactive function. The messages we choose to communicate can perform various interactive and social functions. For example, we can use language to change the way the world is or make things happen. The language provides a means of 
communication, allowing us to share our wishes and desires. Another way in which language fulfills the interactive function relates to the notion of expressivity. Language allows us to express our thoughts and feelings about the world and consider the different mental images evoked by the argumentation. The language also plays a role in how we affect other people in the world and how we make others feel by our choice of words. That is, language can provide information about affect (emotional response). The language we choose to use conveys information about our attitudes concerning others, ourselves, and the situations in which we find ourselves. Language can be used to create scenes or frames of experience, indexing, and even constructing a particular context (Fillmore, 1982). In other words, just by hearing or reading the argumentation, an entire frame is invoked; these guides how we should respond to what follows, what our expectations should be, and so forth [10, P.5-14].

F. van Eemeren and R. Grootendorst distinguish five types of communicative acts, they are assertive - assertion act; commissive - circumstances acceptance act; directive - direction to do something act; expressive - opinion expression act; declarative - new proposal declaration act. In accordance with this division, it is possible to identify the major characteristics necessary for teachers in order to control an argumentative discourse development. They are: ability to convince and assert addressee by means of a language; ability to accept positions and circumstances and reflect it with the help of a language; ability to give clear directions and convince in the necessity to follow them; ability to express (personal) opinion and convince addressee in its relevance; ability to propose new positions using a language. Thus, organizing specialist training according to the mentioned grounds would be beneficial for personal and professional development [11].

According to the modern view on a business communicator and his her role in society, it is not a primary function of information transmission anymore but to become a model of appropriate communicative behavior in various spheres. The importance of argumentative competence in communication, including its intercultural level, cannot be underestimated. That is why the formation of the culture of argumentative discourse is proclaimed to be the key feature of professional development. Obviously, the process of necessary skills formation is to be properly organized and guided by, as proposed, the model of utterances organization. Such a design and differentiation between communicative acts in professional training would certainly be beneficial for both personal and professional growth.

The development of the argumentation culture is seen as the perfect tool for overcoming educational issues and 'curing' the nation. By implementing the concept of argumentative skills development, we will be able to raise the general status of a teacher and equip students with the necessary techniques. Thus, the article is primarily focused on the basic background of argumentative discourse design, which would be beneficial within the educational process. Argumentation, as a scientific category, has been in the focus of investigation interest since ancient times. Today, particular attention to the issue is paid, especially by philosophic, logic, and rhetorical studies. No doubt, each discipline underlines definite peculiar features and determines their approaches to argumentation research. In this sense, the concept of argumentative skills seen as an adequate means for educational purposes, and its awareness might be interpreted as the power to establish rapport with interlocutors and gain a consensus in the process of business communication. If we regard argumentative discourse as an activity in development, two forms of development can be identified. One is enhanced skill in directing the course of critical dialogue to meet the activity's objectives. Other enhanced understanding of the goals of argumentative discourse. To understand the conditions under which argumentative dialogue promotes reasoning, it was necessary to consider people's goals while arguing. In argumentative dialogue, one can distinguish two overlapping but distinct kinds of activity: dispute and deliberation. Moreover, according to the aspect of the discourse, it was necessary to highlight the functions of the language and communicative acts lied under academic communication.

\section{Conclusions}

The results of the post-experimental diagnostics based on mathematical data processing. The dynamics of skills development in experimental and control groups were notably different. The students from experimental groups have demonstrated the improvement of all testing parameters, while the students from control groups have not demonstrated significant improvement in the tested skills. We presented our stages and showed how the teacher could expand the teaching process's guideline model with the help of innovative methods and techniques. Therefore, following the general model's guidelines, the teacher should stay creative, modifying the already set teaching model with his innovations and personal research.

\section{REFERENCES}

[1] Kalbirova T, Karabayeva A, Umirbekova A, Shokaeva K, Bekova D, Anarbek L (2016) Development of cross cultural competence as a factor of cultural - linguistic personality formation. ISJ Theoretical \& Applied Science, 03 (35): 149-153.

[2] Grigorieva V.S. (2007). Discourse as an element of communicative process: Pragmalinguistic and Cognitive aspects. Tambov: TSTU. 
[3] Weston A. (2009). A Rulebook for Arguments. (4 ${ }^{\text {th }}$ ed.). Hackett Publishing Company, Inc.

[4] Houtlosser P. (2001). Points of view. In Eemeren F. (Eds.), Crucial concepts of in argumentation theory. (pp. 34-61). Amsterdam: Amsterdam University Press.

[5] Saez F. (2002). Towards interculturality through language teaching: argumentative discourse. Cause, 25, 103-120.

[6] Chomsky, Noam (1991). Kasher, Asa (ed.). Linguistics and Cognitive Science: Problems and Mysteries. Oxford: Blackwell. p. 50.

[7] Michael Tomasello, Malinda Carpenter, Josep Call, Tanya Behne, Henrike Moll, 2005, « Understanding and sharing intentions: The origins of cultural cognition »: p.721-727 in Behavioral and brain sciences 28(5).

[8] http://www.denstoredanske.dk/Samfund,_jura_og_politik/S prog/Sprogforskeres_biografier/William_Croft.

[9] Langacker, Ronald (2008). Cognitive grammar: a basic introduction. Oxford University Pres.
[10] Evans, Vyvyan (2007). "A Glossary of Cognitive Linguistics". A Glossary of Cognitive Linguistics: P.5-14

[11] F. H. van Eemeren \& R. Grootendorst (2004). A systematic Theory of Argumentation the Pragma-Dialectical Approach.

[12] http://www.youtube.com/watch?v=iGDxCg90Its\&feature= player_embedded

[13] http://www.stanford.edu/dept/CTL/cgi-bin/docs/newsletter/ case_studies.pdf

[14] http://www.crlt.umich.edu/tstrategies/tscbt.php

[15] Aleftina Golovchun, Zhanat Dauletbekova, Raigul Rakhmetova, Aitzhamal Rauandina, Gulzhan Tekesbayeva "Contemporary Methodology of Teaching Kazakh Language in Russian Speaking Groups of Students ", Astra Salvensis, V (2017), Supplement no.1, p. 241-249

[16] Zhumasheva A.Sh., Zhumabaeva Z.E., Sakenov J.Zh., Ismagulova B.H., Sametova F.T., Bazarbaeva A.S. Philological disciplines as means of preparation of students to professional activity. (2014). Life Science Journal 2014; $11(4 s)$. 